\title{
Decision support systems: a framework for re-engaging end-users and improving model uptake
}

\author{
$\underline{\text { E.C. Heagney }}^{\text {a }}$, J.E. Ling ${ }^{a}$, B. Alexander ${ }^{\text {a }}$ and N. Saintilan ${ }^{\text {a }}$ \\ ${ }^{a}$ Office of Environment and Heritage, NSW Department of Premier and Cabinet \\ Email: elizabeth.heagney@environment.nsw.gov.au
}

\begin{abstract}
The Rivers \& Wetlands Unit of the NSW Office of Environment \& Heritage recently commissioned 4 Environmental Decision Support Systems (DSSs) as the culmination of a 3-year \$8.1M multi-disciplinary research program that focused on better use of environmental water in Australia's rivers and wetlands. The program also included a substantial knowledge $\&$ adoption component aimed at effecting uptake of scientific outputs, including a number of presentations and hands-on training workshops focused specifically on DSSs. However, subsequent evaluation indicated that uptake of DSSs remained low, as is typical of many DSSs, and identified significant potential for policy makers and water managers to become disengaged from ongoing model use and development. In the context of environmental water management in Australia, the critical state of river and wetland ecosystems requires that management is underpinned by the best available science, and large and controversial expenditures associated with the purchase of environmental water have brought increased public scrutiny and a heightened requirement for transparent and accountable decision-making. The DSSs produced under this program have been designed specifically to address some of these emerging policy and management requirements, but if they are to fulfill their intended function and feature in future decisions regarding the management of environmental water, it will be necessary to re-invent models and re-engage intended end-users around DSSs and the model development process. We undertook a social research program comprising in-depth interviews with 47 internal and external scientists, policy makers and water managers to identify characteristics of DSS models and the model development process that may present barriers to uptake and individuals' perceptions of science and scientific tools more generally. Primary barriers to uptake for our DSSs related to concerns regarding model accuracy - particularly where models did not adequately incorporate or simulate managers' experiential knowledge or where individuals feared that model outputs from scenario testing might become prescriptive for management - and poor model accessibility - particularly relating to slow data turnaround times that can mean that information is not available to managers when it is required. In order to address these concerns and re-engage stakeholders around DSS models and the model development process, we have developed a confidence-for-purpose framework - where models are required to demonstrate some pre-determined benchmark of data accuracy / reality, set in close consultation with the intended end-users, before they are applied to increasingly complex management functions. Ongoing dialogue established through the process of bench-marking would also allow decisions regarding future investments in next-generation models and/or simplified operational equivalents to be made jointly by model developers and end-users, to achieve a working balance between model accuracy and accessibility - a challenge inherent in all DSSs.
\end{abstract}

Keywords: $\quad$ Decision support systems, model uptake, knowledge and adoption, end-user engagement 


\section{INTRODUCTION}

The Rivers \& Wetlands Unit of the NSW Office of Environment \& Heritage recently commissioned four Environmental Decision Support Systems (DSSs) as the culmination of a three-year $\$ 8.1 \mathrm{M}$ multi-disciplinary research program jointly funded the NSW and Australian governments - the Rivers Environmental Restoration Program (RERP) that focused on better use of environmental water in Australia's rivers and wetlands. The program produced important scientific advances across a range of fields, including inundation and vegetation mapping, fauna surveys, trophic dynamics and hydrological and hydrodynamic modelling, and findings across these fields were integrated into DSSs for four key wetland sites in the Murray-Darling Basin: Narran Lakes, the Gwydir wetlands, the Lowbidgee and the Macquarie Marshes.

DSSs produced under RERP model wetland-scale responses to interacting climate and water management factors (volumes of environmental water, rate and timing of application) and provide comprehensive spatial representation of river and wetland hydrology and habitats. Models run from output files generated by sitespecific hydrologic models (adapted from Integrated Quantity Quality Models developed by the NSW Office of Water), and response variables representing habitat quality for up to 11 key indicator species (vegetation and fauna) are calculated using species response curves and/or Bayesian networks (iCAM, 2010, iCAM, 2011 a,b, SKM/eWater, 2011). Models were developed by external consultants (Sinclair Knight Merz / eWater CRC and Australian National University Enterprise) in consultation with scientists from the Rivers \& Wetlands Unit.

RERP also included a substantial knowledge \& adoption component, and considerable efforts were made to engage policy makers, water practitioners and other stakeholders in an attempt to effect uptake of the suite of scientific outputs produced under the program. Knowledge and adoption initiatives included a number of presentations and hands-on training workshops focused specifically on DSSs. Subsequent evaluation identified variable rates of uptake for RERP outputs, with DSSs faring worse than other scientific products, like inundation and vegetation maps. Low uptake rates for DSSs are not surprising for such early-stage models, but the evaluation process also identified significant potential for policy makers and water managers to become disengaged from ongoing model use and development - a fate that is commonly experienced by DSSs in the environmental field and more generally (Rizzoli and Cuddy, 2005, Giupponi, 2007, Borowski and Hare, 2007). Recent publications have identified a range of reasons for low uptake and use of DSSs, including model complexity (Rizzoli and Cuddy, 2005, Pannell, 2011) a lack of confidence in the accuracy or reality of model outputs (Borowski and Hare, 2007), end-user perceptions about model utility (Van Delden et al., 2007), unrealistic stakeholder expectations and other issues associated with inadequate engagement (Rizzoli and Cuddy, 2005) and inadequate institutional frameworks or support (Pannell, 2011, Eden, 2011). This range of difficulties has led many to question the future of DSSs, and use rates in Australia remain low compared with those in other countries.

While the challenges associated with DSS development and uptake are considerable, there are few alternatives that offer the level of integration and flexibility that are required in a practical management setting. In the context of environmental water management in Australia, the critical state of many river and wetland ecosystems requires that management is underpinned by the best available science, and large and controversial expenditures associated with the purchase of environmental water have brought increased public scrutiny and a heightened requirement for transparent and accountable decision-making. The DSSs produced under RERP have been designed specifically to address some of these emerging management requirements, but if they are to fulfill their intended function and feature in future decisions regarding the management of environmental water, it will be necessary to re-invent models and re-engage intended endusers around DSSs and the model development process.

We have used a combination of qualitative and quantitative research techniques to identify characteristics of DSSs and/or the model development process that have contributed to low rates of uptake for RERP models and present a framework for ongoing model development to improve both the performance of DSSs, and their uptake by policy makers and water managers.

\section{METHODS}

We chose a subset of nine projects completed by the Office of Environment and Heritage under RERP (and/or its immediate predecessor the NSW Wetlands Recovery Program) representing a diverse range of scientific data types (field-based, remotely sensed, lab-based and modelling) and outputs (reports, maps, conceptual models, hydrological and hydrodynamic models and DSSs). Each project was classified using a 
range of variables relating to data confidence, data familiarity, data accessibility, and end-user engagement over the project timeline. Descriptor variables and scores ascribed to each RERP project are provided in Tables 1 and 2 respectively.

Table 1. Project attributes, descriptors score categories or values

\begin{tabular}{|c|c|c|}
\hline Project Attribute & Descriptor & Scores \\
\hline Data confidence & Data type & $\begin{array}{l}\text { 1- observational (field-based or remotely sensed) } \\
\text { 2- laboratory based investigations } \\
\text { 3- modelling }\end{array}$ \\
\hline \multirow{2}{*}{ Data familiarity } & Age of project & Years \\
\hline & Age of output & $\begin{array}{l}\text { Approximate number of years same or similar } \\
\text { outputs have been produced by } \mathrm{OEH}^{*}\end{array}$ \\
\hline \multirow{2}{*}{ Data accessibility } & Deliverable & $\begin{array}{l}\text { 1- map or visual aid } \\
\text { 2- data set } \\
\text { 3- conceptual model or framework }\end{array}$ \\
\hline & Data turnaround time & $\begin{array}{l}\text { 1- immediate } \\
\text { 2- days to weeks } \\
\text { 3- weeks to months }\end{array}$ \\
\hline \multirow{3}{*}{ End-user engagement } & Timing of consultation & First phase of consultation ${ }^{\wedge}$ \\
\hline & Intensity of consultation & No. of consultation phases ${ }^{\wedge}$ \\
\hline & Project leader affiliation & $\begin{array}{l}\text { 1- Rivers \& Wetlands Unit, OEH } \\
\text { 2- External }\end{array}$ \\
\hline
\end{tabular}

*OEH: Office of Environment \& Heritage or its predecessors

$\wedge$ along a project timeline with seven phases: stimulus, concept, planning, data collection, data analysis, data interpretation, delivery of final product

Table 2. Descriptor scores ascribed to selected RERP projects

\begin{tabular}{l}
\hline \\
\end{tabular}


In-depth qualitative interviews were conducted with 47 scientists, policy makers and water practitioners (37 internal to the Office of Environment and Heritage and 15 external) in May 2011, roughly one year after the completion of RERP. Interviews were conducted by an external consultant (Inca Consulting), and used a guided discussion format to assess the level of uptake and use associated with individual projects, and pathways and barriers to the uptake and use of scientific outputs more generally. Projects were classified as either high-or low- uptake based on analysis provided by the consultant, and discriminant function analysis (DFA) was performed to ascertain the relative importance of descriptor variables in effecting uptake and use of scientific outputs. Results from DFA were interpreted with close reference to the qualitative data, issues, and insights into individuals' perceptions and behaviours gained during in-depth interviews.

\section{RESULTS \& DISCUSSION}

DFA identified two axes discriminating high versus low uptake outputs (Figure 1): the first axis ('data confidence / accessibility') was highly correlated with 'data type' $\left(\mathrm{R}^{2}=0.95\right)$ and 'data turnaround time' $\left(\mathrm{R}^{2}=0.83\right)$ and described $44 \%$ of variance; the second axis ('data familiarity') was correlated with 'age of output' $\left(\mathrm{R}^{2}=0.86\right)$ and described a further $29 \%$ of variance. Interviews with scientists and water managers confirmed that low uptake of DSSs relate primarily to issues of data confidence and accessibility. The nature of the barrier(s) to uptake associated with each of these project characteristics is discussed in greater detail below with reference to insights into end-user perceptions gained through qualitative interviews. We also discuss the role that targeted end-user engagement may play in diminishing barriers and effecting better use and uptake of DSSs and other scientific models.

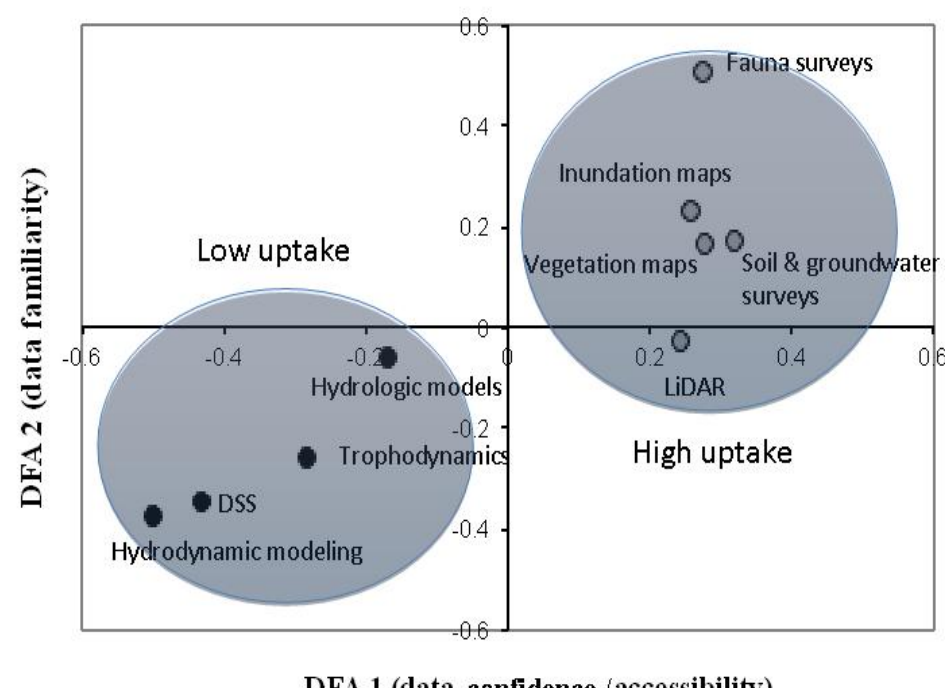

DFA 1 (data confidence /accessibility)

Figure 1.First (x) and second (y) discriminant function axes for high versus low uptake outputs

\subsection{Data confidence}

The high correlation between project uptake and 'data type' suggests that the low uptake and use rates experienced by DSSs are common to modelling projects more broadly. In our study 'data type' has been used as a proxy for data confidence based on perceptions reported from qualitative interviews. Interviewees reported a lower degree of confidence in modelling projects based on the perception that modelling outputs are less accurate than observational data (comprising both field- and remotely-sensed data). Confidence was lowest where outputs were inconsistent with managers' experiential knowledge or expectations (low data 'reality'), or where there was some concern that model outputs might become prescriptive in the decisionmaking process.

Concerns regarding model accuracy or reality have been identified by a range of research groups (e.g. Borowski and Hare, 2007) and raise a number of questions about the way we view and use DSSs and other integrated scientific models. It is true that moving from observational to modelling studies involves the 
addition of new assumptions and/or increased uncertainty associated with model parameters and functions, but in practice, the level of uncertainty associated with integrated models is no greater than that inherent in applying observational findings from a past study to a new management setting. Indeed, the degree of uncertainty associated with integrated models is likely to be lower, on the basis that they generally rely on meta-analysis of multiple observational datasets and are therefore more robust to the influence of timing, site or other study-specific influences than information derived from a single study. However, reluctance to view DSSs as the 'best available science' for input into decision-making remains, and may stem, in part, from an over-reliance on published science or expert opinion from scientists and a tendency for model developers to overlook the vast body of knowledge held by field-based managers. Indeed, managers' experiential knowledge often spans long temporal scales and encompasses a broad range of climatic, environmental and hydrological conditions, and should be harnessed to improve model performance and enhance uptake rates.

Some interviewees reported a reluctance to engage with DSSs based on a fear that model outputs from scenario testing might become prescriptive for management, replacing and removing the human element from the decision-making process. Similar concerns have been identified as a barrier to uptake of DSSs more generally (e.g. Possingham, 2009). However, prescriptive model application is neither likely nor desirable. The DSSs produced under RERP, like many other environmental DSSs, only integrate outcomes for selected response variables, but management is likely to be interested in outcomes for a range of additional variables or species that would need to be considered alongside model outputs. They also deal exclusively with biophysical data, so outputs will always need to be weighed against economic and social and logistical concerns. Moreover, DSSs can help to inform decision-making by performing a range of valuable functions (e.g. sensitivity analyses, identification of unintended consequences) that require more moderate levels of data confidence, and a failure to engage DSSs precludes these valuable inputs to management.

\subsection{Data accessibility}

Data accessibility, and in particular data turnaround time, was another important factor affecting uptake. DSSs and other models produced under RERP are not currently available (or suitable) for policy makers and managers to use independently, and can only be accessed via a request (however informal) to scientists from the Rivers \& Wetlands Unit to model scenarios of interest. In the absence of a dedicated staff member to respond to these requests, any modelling undertaken for managers or other end-users will necessarily experience some delay. In-depth interviews revealed that managers generally access science for a specific purpose (annual planning, watering event or reporting requirement) so delays associated with modelling may mean that outputs are not available to managers when they are required. This would undoubtedly be the case for tactical decisions that are made on very short timeframes during watering events. In order to achieve model uptake, outputs need to be delivered to managers more quickly either by providing field managers with access to modelling support staff when they might be required (i.e. during the watering season) or by providing operational outputs (e.g. scenario libraries or simplified model interfaces) that can be used independently by policy makers and managers without placing an unreasonable demand on their time.

\subsection{A role for end-user engagement?}

Our analysis highlights a range of project and/or model characteristics that have a substantial impact on the uptake and use of scientific outputs, lending support to the idea that uptake and use are not achieved through more or better communication alone (Borowski and Hare, 2007) - with three caveats:

1. Communication must be sufficient to effect awareness of scientific outputs and an understanding of their potential applications. Interviews indicated that this was achieved for all RERP projects

2. Familiarity with research methodologies and outputs, which had a secondary effect on uptake, is enhanced by effective and ongoing consultation with policy makers and managers

3. The accuracy and accessibility of DSSs are dependent on effective two-way communication to ensure that managers' experiential knowledge and preferences are adequately represented.

In the case of DSSs produced under RERP, in-depth interviews suggested that policy makers and managers, who are the intended end-users, are at risk of becoming disengaged from ongoing model development $-\mathrm{a}$ situation that is likely to be true for most DSSs that have not met managers' needs or expectations. Effective communication and strategic end-user engagement are key to overcoming the main barriers to uptake identified in this study. Effective communication regarding the role of DSSs in integrating the best available 
science for a range of decision making purposes may help to overcome barriers associated with data accuracy and confidence; and effective consultation during the initial and ongoing model development phases may lead to DSSs that are more consistent with managers' preferences for accessing scientific information, and thereby experience better rates of uptake. To this end we have developed a framework for end-user engagement and model uptake that can be applied to DSS projects during design and ongoing development phases to encourage or improve end-user involvement and subsequent uptake (Section 3.4). The framework is consistent with published literature which suggests that uptake of scientific outputs is enhanced when engagement involves repeated interactions with key stakeholders, attempts to make science and scientific models more transparent, and provides genuine opportunities for collaboration (Eden, 2011).

\subsection{A framework for end-user engagement and model uptake}

Uptake of DSSs could be improved using a confidence-for-purpose framework - where models are required to demonstrate some pre-determined benchmark of data accuracy / reality, set in close consultation with the intended end-users, before they are applied to increasingly complex management functions (Figure 2). Ongoing dialogue established through the process of bench-marking would also allow decisions regarding future investments in next-generation models and/or simplified operational equivalents to be made jointly by model developers and end-users, to achieve a working balance between model accuracy and accessibility $-\mathrm{a}$ challenge inherent in all DSSs (e.g. Salewicz and Nakayama, 2004, Rizzoli and Cuddy, 2005, Argent et al., 2009) -and improving model utility and uptake. Employing an overarching framework also provides a 'shared vision' between model developers and end-users and clarity about project goals and collaborators' responsibilities (Herron and Cuddy, 2008) and builds trust - an important precursor to model adoption.

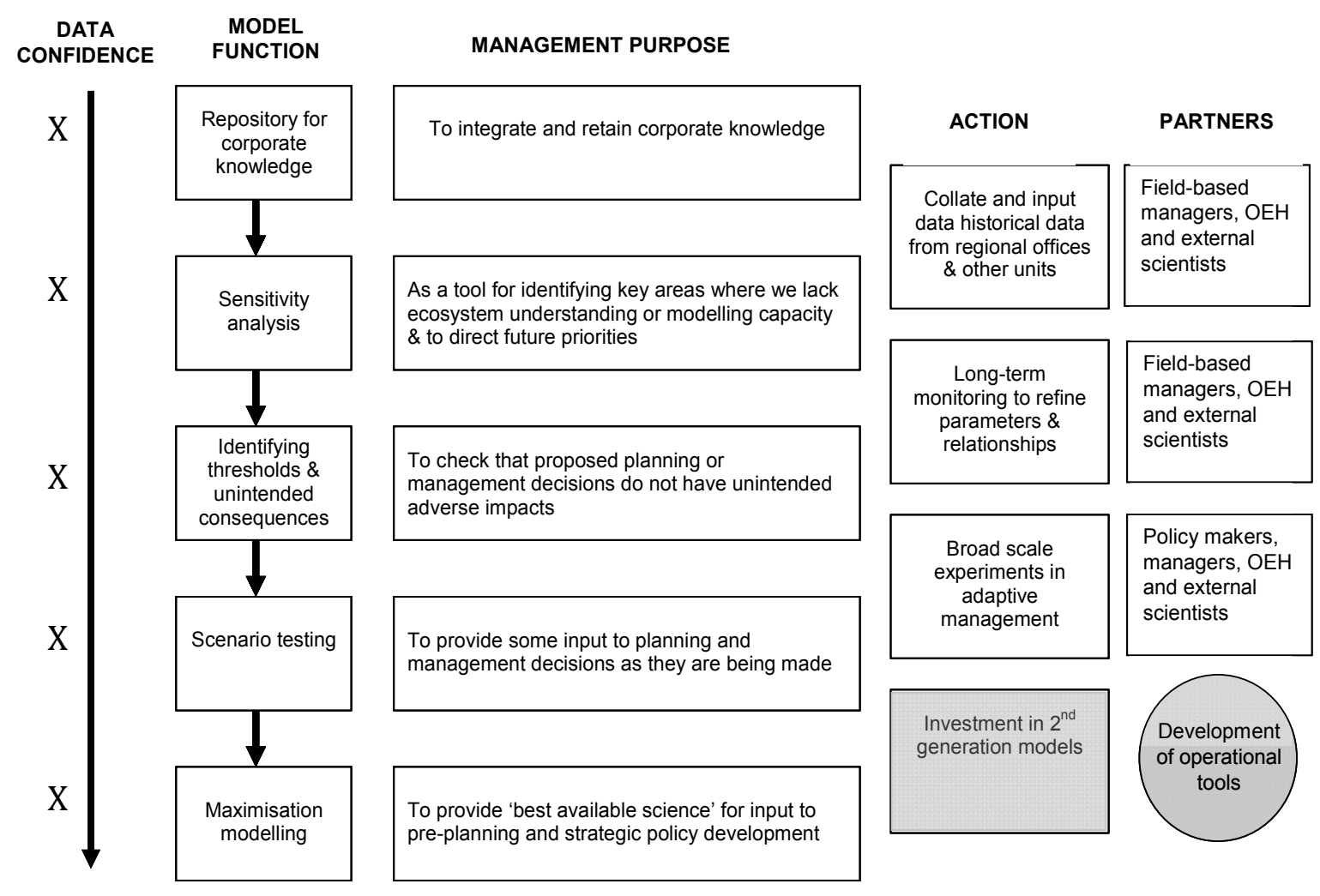

Figure 2. A confidence-for-purpose framework to link model function with data accuracy and guide engagement of model end-users during ongoing model development.

$\mathrm{X}$ denotes points where pre-determined benchmarks of data accuracy or reality would be set, with an increasing requirement for accuracy stepping through model functions from top to bottom. Grey shading indicates actions that are not fixed along the project timeline.

OEH: Office of Environment \& Heritage or its predecessors 


\section{CONCLUSIONS AND RECOMMENDATIONS}

Most DSSs suffer low uptake. In the absence of any clear alternative for incorporating the best available science into decisions regarding our increasingly rare and degraded natural ecosystems, overcoming barriers to uptake is crucial. Given that the main uptake barriers identified in our research relate to data confidence and accessibility, we suggest that there is an onus on model developers to manage and meet end-users' expectations around these model characteristics, and recommend a confidence-for-purpose framework to reengage model end-users and encourage uptake.

Our future research in this field will be focused around quantifying the level of data confidence that scientists and managers consider suitable for the range of model functions outlined in Figure 2, characterising preferences for trade-offs between data accuracy and accessibility, and continuing evaluation of the uptake and use of DSSs and other scientific outputs produced under RERP in response to ongoing efforts around end-user re-engagement.

\section{ACKNOWLEDGEMENTS}

The Rivers Environmental Restoration Program (RERP) was supported by the NSW Government and the Australian Government's Water for the Future - Water Smart Australia Program. RERP aims to arrest the decline of wetlands through water recovery, effective management of environmental water and the sustainable management of our wetlands. The evaluation of uptake and use of science produced under RERP was funded by the National Water Commission. In-depth interviews that form the basis of many of the insights presented in this paper were undertaken by Inca Consulting. We thank all those who agreed to be interviewed for this study.

\section{REFERENCES}

Argent, R.M., Perraud, J.-M., Rahman, J.M., Grayson, R.B., and Podger, G.M. (2009). A new approach to water quality monitoring and environmental decision support systems. Environmental Modelling \& Software 24, 809-818.

Borowski, I., and Hare, M. (2007). Exploring the gap between water managers and researchers: difficulties of model-based tools to support practical water management. Water Resource Management 21, 1049-1074.

Eden, S. (2011).Lessons on the generation of usable science from an assessment of decision support practices. Environmental Science \& Policy 14, 11-19.

Giupponi, C. (2007). Decision support for implementing the European water framework directive: the MULINO approach. Environmental Modelling \& Software 22, 248-258.

SKM/eWater (2011). Decision Support System for the Lowbidgee Wetland. Final report prepared for the Rivers Environmental Restoration Program, NSW Office of Environment \& Heritage.

Herron, N.F. and Cuddy, S.M. (2007). Better DSS Design through Effective Engagement of External and Internal Stakeholders. Proceedings of the International Congress on Modelling and Simulation MODSIM2007 Modelling and Simulation Society of Australia and New Zealand Inc. (MSSANZ), Oxley, L., Kulasiri, D. (Eds), Christchurch, NZ. , 650-656.

iCAM (2010). Decision Support System for the Gwydir Wetlands - Final Report. iCAM, The Fenner School of Environment and Society, Australian National University, Canberra.

iCAM (2011a). Decision Support System for the Macquarie Marshes- Final Report. iCAM, The Fenner School of Environment and Society, Australian National University, Canberra.

iCAM (2011b). Decision Support System for the Narran Lakes - Final Report. iCAM, The Fenner School of Environment and Society, Australian National University, Canberra.

Inca Consulting (2011). Uptake and use of science-based knowledge and tools in water and wetland management A Final report on key findings. Report to the NSW Office of Environment and Heritage.

Pannell, D. (2011). Why don't environmental managers use decision frameworks? Decision Point 54, 2.

Possingham, H. (2009). Hughbris of the decision theorists: If decision theory s so good why don't more people use it? Decision Point 27, 2-3.

Rizzoli, A.E., and Cuddy, S.M. (2005). Do DSSs fulfill their promises? Success and failure of decision support systems for integrated water resource management. Venice 6-7 October 2005.

Salewicz, K.A., Nakayama, M. (2004). Development of a web-based decision support system (DSS) for managing large international rivers. Global Environmental Change 14, 25-37.

Van Delden, H. Luja, P. Engelen, G. (2007). Integration of multi-scale dynamic spatial models of socioeconomic and physical processes for river basin management. Environmental Modelling \& Software 22, 223-228. 